\title{
Self-reported dietary intake of youth with recent onset of type 2 diabetes: Results from the TODAY study
}

\author{
Linda Delahanty, MS, RD [Director of Nutrition and Behavioral Research], \\ Massachusetts General Hospital; 50 Staniford Street, Suite 340, Boston, MA 02114, USA \\ Idelahanty@partners.org
}

\begin{abstract}
Andrea Kriska, PhD [Professor],
Department of Epidemiology, Children's Hospital of Pittsburgh of UPMC; One Children's Hospital Drive 4401, Penn Avenue Faculty Pavilion, Floor 6 Pittsburgh, PA 15224, USA;

KriskaA@edc.pitt.edu

Sharon Edelstein, ScM [Senior Research Scientist], George Washington University Biostatistics Center; 6110 Executive Boulevard Suite 750, Rockville, MD 20852, USA; sharone@biostat.bsc.gwu.edu

Nancy Amodei, PhD [Clinical Professor], Department of Pediatrics University of Texas Health Science Center at San Antonio; 7703 Floyd Curl Drive, San Antonio, TX 78229, USA; AMODEI@uthscsa.edu
\end{abstract}

Jennifer Chadwick, BS [Native American Program Coordinator], OU Children's Diabetes Center TODAY Study; 1200 NE Phillips Suite 4500, Oklahoma City, OK 73104, USA; jennifer-chadwick@ouhsc.edu

\section{Kenneth Copeland, MD [Jonas Professor and Section Chief of Pediatric Diabetes/ Endocrinology] [Vice Chairman of the Department of Pediatrics], University of Oklahoma Health Sciences Center TODAY Study OUCPB; 1200 NE Phillips Suite 4500, Oklahoma City, OK 73104, USA; Kenneth-Copeland@ouhsc.edu \\ Bryan Galvin, MS [Research Coordinator], \\ UPMC Department of Orthopaedic Surgery; 3200 South Water Street, Pittsburgh PA, 15203 USA;Bryan.Galvin@chp.edu}

\author{
Laure El ghormli, MS [Research Scientist], \\ George Washington University Biostatistics Center; 6110 Executive Boulevard Suite 750, \\ Rockville, MD 20852 USA; elghorml@biostat.bsc.gwu.edu
}

\author{
Morey Haymond, MD [Professor of Pediatrics], \\ Baylor College of Medicine Children's Nutrition Research Center; 1100 Bates Street, Houston, \\ Texas 77030, USA; mhaymond@bcm.edu
}

\section{Megan Kelsey, MD [Assistant Professor of Pediatric Endocrinology],}

\footnotetext{
() 2013 Academy of Nutrition and Dietetics. Published by Elsevier Inc. All rights reserved.

Corresponding author: Laure El ghormli, George Washington University Biostatistics Center, 6110 Executive Blvd Suite 750, Rockville, MD 20853, 301-881-9260 (p), 301-881-3767 (f), elghorml@ biostat.bsc.gwu.edu.

Publisher's Disclaimer: This is a PDF file of an unedited manuscript that has been accepted for publication. As a service to our customers we are providing this early version of the manuscript. The manuscript will undergo copyediting, typesetting, and review of the resulting proof before it is published in its final citable form. Please note that during the production process errors may be discovered which could affect the content, and all legal disclaimers that apply to the journal pertain.

Current affiliation: Chad Lassiter MSW, President, Black Men at Penn, University of Pennsylvania; 3701 Locust Walk, Philadelphia, PM 19104, USA; Children's Hospital of Philadelphia 34th St. and Civic Center Blvd. Philadelphia, PA 19104
} 
Children's Hospital Colorado; 13123 East 16th Avenue B265, Aurora, CO 80045, USA;

Megan.Kelsey@childrenscolorado.org

Chad Lassiter, MSW [Behavioral Interventionist],

Children's Hospital of Philadelphia 34th St. and Civic Center Blvd. Philadelphia, PA 19104

Kerry Milaszewski, BS, RN [Pediatric Diabetes Nurse Educator],

Joslin Diabetes Center, One Joslin Place; Boston, MA 02215, USA;

kerry.milaszewski@joslin.harvard.edu

Amy Syme, BS [Research Coordinator III], and

Yale University Department of Pediatric Endocrinology; 2 Church St South Suite 201, New

Haven, CT 06519, USA; amysyme@gmail.com

Elizabeth Mayer-Davis, PhD [Professor]

Department of Nutrition, University of North Carolina Chapel Hill; 2211 McGavran Greenberg, CB

7461, Chapel Hill, NC 27599-7461, USA; ejmayer_davis@unc.edu

\section{Abstract}

Despite the widely recognized importance of diet in managing diabetes, few studies have documented usual dietary intake in youth with type 2 diabetes (T2D). The objectives of this study were to assess dietary intake among a large, ethnically diverse cohort of youth with T2D and compare intake to current recommendations. Treatment Options for type 2 Diabetes in Adolescents and Youth (TODAY) is a multi-center randomized clinical trial of 699 youth aged 10-17. At baseline, following a run-in period that included standard diabetes education, diet was assessed using a food frequency questionnaire between 2004 and 2009. Analysis of variance and non-parametric tests were used to compare mean and median nutrient intakes; logistic regression was used to compare the odds of meeting pre-defined dietary intake recommendation cut points between subgroups of age, sex and race-ethnicity. Percent of energy from saturated fat was consistently $13-14 \%$ across all subgroups - substantially exceeding national recommendations. Overall, only $12 \%$ of youth met Healthy People (HP) 2010 guidelines of $<10 \%$ saturated fat and only $1 \%$ of youth met American Diabetes Association recommendations of $<7 \%$ saturated fat. Dietary intake fell substantially below other HP 2010 targets; only 3\% met calcium intake goals, $11 \%$ met fruit consumption goals, $5 \%$ met vegetable consumption goals, and $67 \%$ met grain intake goals. Overall, dietary intake in this large cohort of youth with T2D fell substantially short of recommendations, in ways that were consistent by sex, age, and race-ethnicity. The data suggest a critical need for better approaches to improve dietary intake of these youth.

\section{Keywords}

food/nutrient intake; diet intake assessment; type 2 diabetes; adolescence; obesity/overweight

The American Diabetes Association (ADA) nutrition recommendations for youth with type 2 diabetes (T2D) emphasize the importance of meeting targets for glycemic control, blood pressure and lipids and the role of medical nutrition therapy (MNT) in achieving these goals $^{1,2}$. MNT recommendations focus on cessation of excessive weight gain while promoting normal linear growth through reduced intake of high fat, high energy dense foods, saturated fat, and cholesterol together with increased physical activity. The ADA models food intake recommendations for people with T2D on The Dietary Guidelines for Americans ${ }^{3,4,5}$ and Institute of Medicine (IOM) Dietary Reference Intakes ${ }^{6,7}$, along with evidence-based nutrition research conducted in study participants with T2D. To date, most reports describing youth with and without diabetes indicate intakes of higher than 
recommended levels of total fat and saturated fat, with fewer than $50 \%$ of youth meeting recommendations for calcium, fiber, fruits and vegetables ${ }^{8-11}$.

Despite the widely recognized importance of diet in management of diabetes, few studies to date have documented usual dietary intake in such a large, diverse sample of youth with

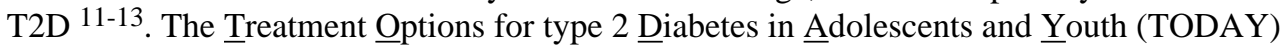
trial provides the opportunity to assess dietary intake among a large, ethnically and regionally diverse cohort of youth with recently diagnosed T2D after participation in a standard diabetes education (SDE) program. This report a) describes estimated intake of nutrients and foods in the TODAY cohort according to age, sex and race-ethnicity and, b) compares intake to current recommendations and to similarly aged non-diabetic populations, and compares the odds of meeting these recommendations between subgroups. It was hypothesized that most youth would not meet nutrition recommendations regardless of age, sex or ethnicity.

\section{Methods}

The TODAY trial is a randomized, double-blind, parallel-group clinical trial designed to evaluate the relative efficacy and safety of three treatments for T2D in youth: metformin alone, metformin plus rosiglitazone, or metformin plus intensive lifestyle intervention ${ }^{14,15}$. The primary objective is to compare the three treatment arms on time to treatment failure, defined as loss of glycemic control (either a hemoglobin A1C of $8 \%$ or greater over a 6month period or inability to wean from temporary insulin therapy within 3 months after metabolic decompensation).

A detailed description of TODAY study methods is published elsewhere ${ }^{14}$. Following individual institutional IRB approval, participants were recruited at 15 clinical centers and provided informed consent; minor children confirmed assent according to local guidelines. Participants were 10-17 years old, with less than two years of T2D, and a BMI 285 th percentile at time of diagnosis or at screening.

Prior to randomization, a run-in period was performed to ensure that participants were able to tolerate therapy with metformin, accomplish mastery of a SDE curriculum and demonstrate ability to adhere to study requirements for pill taking and visit attendance. The SDE curriculum included dietary strategies to reduce calorie intake to avoid excessive weight gain and maintain linear growth in those still growing and to promote weight loss in youth who had stopped growing ${ }^{14,16}$. The SDE program ${ }^{14,16}$ was designed specifically for TODAY participants to provide diabetes knowledge and self-management skills with the aim of all participants having equivalent baseline diabetes knowledge at time of randomization; only $1 \%$ of youth did not proceed to randomization due to inability to master $\mathrm{SDE}^{17}$. Youth who successfully completed the run-in period were randomized to one of three treatment arms and entered the main clinical trial and completed baseline measures.

\section{Assessment of Dietary Intake}

At baseline, following a run-in period that included SDE, diet was assessed using a food frequency questionnaire (FFQ) between 2004 and 2009. Semi-quantitative FFQ methodology was chosen for use in the TODAY trial based on its low cost and reduced participant burden compared to multiple 24 hour dietary recalls. Although FFQs provide less specific information then 24 hour dietary recalls, national surveys such as the National Health Interview Survey ${ }^{18}$ and other major national studies have similarly used FFQ data to describe dietary intake in the study population ${ }^{11,19,20}$; FFQs have also detected significant change in dietary intake over time in the context of clinical trials ${ }^{19}$. FFQs are known to underestimate total energy intake overall, particularly with increasing BMI in both children 
and adults, but do well when examining relative quantities such as percent intake of various nutrients $9-11,21$.

The TODAY FFQ was a modified version of the SEARCH for Diabetes in Youth FFQ ${ }^{11}$ which was derived from the Block Kids' Food Questionnaire ${ }^{22}$. Psychometric evaluation of the Block Kids' Food Questionnaire, which included test-retest reliability and validation against two 24-hour dietary recalls, was conducted in a sample of children as young as 8 years old, including African American youth living in a low-income neighborhood 22 and in an ethnically diverse cohort of youth aged 10-17 years ${ }^{23}$. The SEARCH FFQ included additional food lines based on items commonly consumed by participating minority ethnic and racial groups. The TODAY FFQ was further modified to add an option for youth to report extra-large portion sizes e.g., to allow participants to identify the bigger size choices available in fast food restaurants. The original Block Kids' Food Questionnaire included about 75 food lines; the TODAY FFQ included about 100 food lines. These additions were based primarily on foods identified for inclusion for the SEARCH Study and the Diabetes Prevention Program (DPP) that have similar participant diversity ${ }^{11,19}$. For each line item, participants were asked if the food item was consumed in the past week ("yes/no") and if yes, how many days, and average portion. Portion size was queried for each line item either as a number or as "very small", "small", "medium", "large", or "very large" relative to pictures of food in bowls or plates provided with the form. Other modifications included a small number of questions designed to understand more fully whether the period of recall (one week) reflected "usual" intake for the individual, use of dietary supplements, use of low-fat products and frequency of eating out. These modifications did not change the core structure or content of the Block Kids FFQ instrument, which is designed to permit adding items relevant to each study. The nutrient and portion-size databases for this instrument were modified from SEARCH FFQ databases, using the Nutrition Data System for Research (database 3 version 4.05/33, 2002, Nutrition Coordinating Center, University of Minnesota, Minneapolis) and industry sources.

\section{Quality Control}

Certified research staff administered the FFQ via interview with direct entry of participant responses into a computer, using software provided by University of South Carolina Diet Assessment Center (DAC). The FFQs were edited initially at clinical sites, and additional editing and quality control checks (e.g., for internal consistency and range) were conducted at DAC using edit checks based on the National Cancer Institute Health Habits and History Questionnaire HHHQ/DietSys program (version 4.01) followed by staff review of FFQs in which a food or nutrient value was found to be extreme.

\section{Anthropometric Measures}

All anthropometric measures were taken with youth wearing lightweight clothing and without shoes by certified research staff. A single height measurement was recorded using a clinical stadiometer. Stadiometer brand varied by site but a single stadiometer was used to measure height at each site. Weight was measured in duplicate using a Seca scale (model 882; Seca USA, Hanover, MA) with a third measurement made only if the first measurements differed by more than $0.2 \mathrm{~kg}$. Height was measured to the nearest $0.1 \mathrm{~cm}$ and weight was measured to the nearest $0.1 \mathrm{~kg}$. BMI was calculated as $\mathrm{kg} / \mathrm{m}^{2}$.

\section{Statistical Methods}

Ethnicity was determined by self report. For data analysis, 25 (3.6\%) participants who reported belonging to more than one racial group were assigned to a racial-ethnic group according to the following priority of risk for T2D in youth: American Indian (AI) greater than Hispanic greater than Non-Hispanic Black (NHB) greater than Non-Hispanic White 
$(\mathrm{NHW})^{24}$. Tests performed excluded the AI group due to small numbers. Data are reported as mean, standard deviation, median, $25^{\text {th }}$ and $75^{\text {th }}$ percentile of the distribution or percents as appropriate. Analysis of variance and non-parametric tests were used to compare mean and median nutrient intakes and logistic regression was used to compare the odds (OR and 95\% CI) of meeting pre-defined cut points, by age, sex and self-reported race/ethnic group. In order not to confuse calorie requirements for weight maintenance with calorie requirements for weight loss in these overweight and obese youth, servings from each food group were not based on energy intake but rather the lower end of the recommended range of servings per day for the various food groups as described in 2000 Dietary Guidelines ${ }^{3,4}$. Using the minimum standard as a basis for determining the percent of youth meeting the dietary guidelines was appropriate to our goals of not overstating the inadequacy of the diet and ease of comparison to earlier reports ${ }^{11}, 19,20$. Due to the number of statistical tests conducted, $\mathrm{a}=.015$ was used to test for statistical significance. Analyses were conducted using the Statistical Analysis System (version 9.2, SAS Institute Inc, Cary, NC).

\section{Results and Discussion}

Baseline characteristics of the randomized participants have been reported ${ }^{17}$. Briefly, the study population is largely of minority racial-ethnic background, with $19.6 \% \mathrm{NHW}, 41.1 \%$ Hispanics, 31.5\% NHB, 6.1\% AI and 1.7\% Asian, and comes from families of low education level and annual household income. An annual household income of less than $\$ 25,000$ was reported by $41.5 \%$ of parent/guardians; $26 \%$ of parent/guardians reporting less than $12^{\text {th }}$ grade education. Overall, participants were evenly distributed between younger (10-14 years) and older (15-18 years) adolescents and there were more female than male participants (64.9\% vs. $35.1 \%)$. The majority were obese $(86.9 \%)$ and $10.7 \%$ were overweight but not obese.

Of 699 TODAY cohort participants, 672 baseline FFQs were received; 24 were excluded for the following reasons: 1$)$ excess calories $(>8000)(\mathrm{n}=0) ; 2)$ reporting $<3$ average foods per day or reporting eating 'a lot more/a lot less' than usual' $(n=18) ; 3)$ reporting between 3 and 3.5 foods per day and having inconsistent responses to cold cereal quality control questions $(\mathrm{n}=3)$; 4) forms for which the values were 0.5 sex-specific standard deviations or more from the nearest neighbor for highest calories among males and females separately $(n=2)$; and 5) comment from interviewer $(n=1)$. Therefore $3.6 \%$ of forms were excluded in the final data set and nutrition analysis for this paper was conducted using FFQ data from 648 TODAY participants. Of these youth, $91 \%$ reported that what they ate was typical of usual eating habits and 9\% reported that what they ate was 'a little more" or "a little less than usual."

\section{Baseline Dietary Intake}

In a typical week, most youth (88\%) reported eating 3 to 5 times per day; $31 \%$ ate the school breakfast or lunch 4-5 times per week. In addition, 33\% of youth reported eating fast food once per week and another $31 \%$ ate fast food 2 or more times per week; $33 \%$ reported eating out at other types of restaurants at least once per week. There were no significant differences by sex or by racial ethnic group in the reported frequency of eating out at fast food or other types of restaurants at least once per week (data not shown).

Reported nutrient intake and food group consumption patterns for girls and boys are shown in Table 1. Overall, youth reported diets that were low in calories, high in total and saturated fat, low in fiber, fruits, vegetables and sweetened drinks, and high in grains. Compared to girls, boys reported more calories, protein, cholesterol, calcium, magnesium and iron as evidenced through more dairy and meat, poultry and fish consumption. There were no significant differences in median macronutrient intake between boys aged 10 to 14 years and 
boys aged 15 to 18 years. However, girls aged 15 to 18 years reported higher median caloric intake (1197 kcal vs $1073 \mathrm{kcal}, \mathrm{p}=.012)$, and more dietary fiber (9.0 g vs $7.7 \mathrm{~g}, \mathrm{p}=.003)$, vitamin C (56 mg vs $44 \mathrm{mg}, \mathrm{p}=.005$ ), vitamin $\mathrm{E}$ ( $4.0 \mathrm{mg}$ vs $3.3 \mathrm{mg}$ a-tocopherol, $\mathrm{p}<.001$ ) and magnesium (151 mg vs $132 \mathrm{mg}, \mathrm{p}=.008$ ) than girls aged 10-14 years. In terms of food group servings per day, the only differences between older and younger boys were that

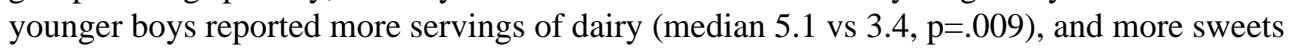
and desserts (median 1.2 vs $0.6, \mathrm{p}=.011$ ). Younger girls reported less daily servings of vegetables ( 0.7 vs $0.9, \mathrm{p}=.008$ ), more daily servings of grains (median 9.0 vs $7.3, \mathrm{p}=.007$ ), and less sweetened drinks (median 0.0 vs $0.1, \mathrm{p}=.001$ ).

Table 2 compares reported nutrient intake and food group consumption patterns by race/ ethnicity. All racial/ethnic groups reported high fat and saturated fat intakes, low dietary fiber, fruit, vegetable and sweetened drink consumption and high intake of grains. Total fat, saturated fat, monounsaturated fat, polyunsaturated fat, vitamin E, dairy intake, fats and oils and sweetened drink consumption were significantly different among ethnic groups. AI youth had the highest and Hispanic youth the lowest percent saturated fat intake. Dietary intake patterns for girls and boys by ethnic group were similar to the overall patterns in Table 2 except that, when girls and boys were considered separately, there were no significant differences in saturated fat intake, vitamin E intake or fats and oils among racial/ ethnic groups. Food group consumption patterns for girls by race/ethnicity were similar to the overall results in Table 2 in terms of sweetened drinks; however, dairy consumption did not reach statistical significance. There were no significant differences in daily food group servings among boys by ethnicity.

Table 3 shows current nutrition recommendations for youth with diabetes based on ADA, American Heart Association (AHA), IOM, FGP and Healthy People (HP) 2010 guidelines, ${ }^{1-7,25,26}$ the percent of youth who met dietary recommendations at baseline after completion of SDE by sex and race-ethnicity, and the odds of compliance between subgroups. Overall, very few youth with T2D met nutrition recommendations for total fat, saturated fat, calcium, fruit and vegetable intake. Girls were less likely to meet goals for daily servings of dairy and meat; however, they were more likely to meet goals for cholesterol intake. There were no significant differences in percent who met dietary recommendations among ethnic groups except that Hispanics were more likely than NHW to meet goals for the percent calories from fat and saturated fat, but were less likely than NHW to meet the $<200 \mathrm{mg}$ cholesterol intake goal. NHB were less likely than Hispanics and NHW to meet the dairy goal.

The TODAY study represents the largest and most ethnically diverse group of adolescents and youth with T2D ever described. Despite participating in a SDE program that focused on weight loss and a healthful diet targeting this demographic ${ }^{16}$, the reported dietary intake of these youth with T2D fell substantially short of national nutrition recommendations irrespective of gender, age and race/ethnicity. The percent of calories from total fat and saturated fat remained higher than recommendations in both younger and older girls and boys and in all ethnic groups. Overall, only $10 \%$ and $12 \%$ of youth met the $\leq 30 \%$ total fat and $\leq 10 \%$ saturated fat recommendations, respectively. Furthermore, a pattern of low intake of dietary calcium, fiber, fruits and vegetables and high intake of grains was evident in each of these groups. When compared to HP 2010 guidelines, this cohort of youth fell significantly below dietary targets; only $3 \%$ met calcium intake goals, $11 \%$ met fruit consumption goals, $5 \%$ met vegetable consumption goals and $67 \%$ met grain intake goals.

Intake of total fat and saturated fat in TODAY participants exceeded the average intake reported for a broad population of overweight and non-overweight similarly aged non- 
diabetic American youth in both girls and boys and for NHB and Hispanics (4-6\% higher and $1-2 \%$ higher, respectively) ${ }^{9}$.

The SEARCH study, which used a similar FFQ instrument to assess dietary intake in youth aged 10-14 and aged 15 and older with either type 1 or T2D of at least 1 year's duration, is an appropriate comparison group for intake of some nutrients ${ }^{11}$. Dietary fat intake among youth with T2D in SEARCH ( $\mathrm{n}=186)$ was $37-38 \%$ and saturated fat intake was $13-14 \%$, very similar to the TODAY cohort. The pattern of low intake of fruits and vegetables was also apparent in SEARCH. The TODAY cohort was heavier than the youth with T2D in the SEARCH cohort, and reported consuming about three times the amount of grains and dairy and double the amount of desserts and sweets compared to the SEARCH group.

Of great concern is the observation that only $12 \%$ of TODAY youth met the IOM and HP 2010 recommendation of $<10 \%$ total calories as saturated fat, and only $1 \%$ met the more stringent $\mathrm{ADA} / \mathrm{AHA}$ recommendation of $<7 \%$. By comparison, in a large population based sample of urban youth aged 11-18 years, in which $13 \%$ were obese and $33 \%$ were overweight, $45 \%$ of girls and $36 \%$ of boys met the $10 \%$ saturated fat intake goal while $46 \%$ of girls and $45 \%$ of boys met fruit consumption goals and $18 \%$ of girls and $16 \%$ of boys met vegetable consumption goals ${ }^{10}$. It is possible that reducing saturated fat intake to recommended targets is more challenging for patients with T2D due to the common focus on carbohydrate counting and reduced sweets which may reinforce an existing pattern of eating less fruits and vegetables and shift eating habits toward greater consumption of low carbohydrate foods such as cheese and meats which are also high in fat, cholesterol and saturated fat.

High fat intake during youth is associated with increased risk for heart disease in adulthood ${ }^{27}$ and low calcium intake leads to low bone density in adolescents and possible osteoporosis in later life ${ }^{28}$. Fruits and vegetables are high in dietary fiber, low in energy density, high in antioxidants and other phytochemicals, and have important implications for management of weight, blood pressure and lipids. A recent study found that a pattern of low consumption of fruits and vegetables is related to arterial stiffness in young adulthood ${ }^{29}$.

Failure to meet nutritional standards in this population is likely underestimated as dietary assessment was completed after SDE and the run-in process which may have biased the cohort by selecting youth who were more highly motivated or interested in healthier behaviors and outcomes. Almost all of AI youth $(n=40)$ in this report originated from a single, predominantly rural state (Oklahoma). Thus, it is possible that effects attributed to AI ethnicity are a result of rural living and available dietary options rather than dietary selections specific to AI youth. Additional research on dietary habits of AI youth is warranted.

The absolute calorie intake was lower than would be anticipated based on the SEARCH findings, which may be related to several factors. The low caloric intake in part may reflect dietary changes or increased socially desirable responses resulting from dietary education provided to the TODAY cohort during the run-in period, whereas the SEARCH participants had no educational intervention. Underestimation of absolute calorie intake may occur, especially in obese children when captured by the FFQ instrument ${ }^{9,11,23}$. Assuming nondifferential under-reporting, in general, such under-reporting of overall intake would bias the findings towards over-estimation of inadequate intake in terms of consumption of foods (e.g., number of servings of fruits and vegetables). Differential under-reporting due to obesity is unlikely to create any additional bias in our data because, by design, all participants were overweight or obese. Adequacy expressed in terms of energy density (e.g., percent of kcal from saturated) fat would not be biased. There could also be some degree of 
under-estimation of inadequacy due to bias related to social desirability such that reported intake could be "healthier" than actual intake.

Nevertheless, the evaluation of food and nutrient intake demonstrates that overall these youth with T2D are not meeting recommended food and nutrient intake guidelines and are consuming diets that may exacerbate cardiovascular and other disease risks. In addition, it is likely that the nutritional gaps identified in the current study underestimate the true nutritional deficits typical of youth with recent onset T2D since the food and nutrient intake for youth who failed the run-in period were not included in the present analyses.

\section{Conclusions}

Changing nutrition and lifestyle habits is often challenging due to a variety of individual, environmental, physiological, cultural and social factors and most families need assistance in overcoming these barriers to dietary change. At the time of entry into TODAY, the diet quality of participants was very poor, with remarkably high intake of saturated fat and very low fruit and vegetable intake. The TODAY study will provide an opportunity to evaluate the incremental impact of a long-term evidence-based nutrition and lifestyle intervention ${ }^{15}$ on dietary intake and health outcomes of these high risk youth with T2D.

\section{References}

1. American Diabetes Association. Type 2 diabetes in children and adolescents. Diabetes Care. 2000; 23(3):381-389. [PubMed: 10868870]

2. Bantle JP, Wylie-Rosett J, Albright AL, et al. Nutrition recommendations and interventions for diabetes: A position statement of the American Diabetes Association. Diabetes Care. 2008; 31(Suppl. 1):S61-S78. [PubMed: 18165339]

3. US Department of Health and Human Services, US Department of Agriculture. Dietary Guidelines for Americans, 2000. US Department of Health and Human Services, US Department of Agriculture; Washington, DC: 2000.

4. The Food Guide Pyramid. US Department of Agriculture Center for Nutrition Policy and Promotion; Washington, DC: 2000. Home and Garden Bulletin No. 252

5. U.S. Department of Agriculture and U.S. Department of Health and Human. Dietary Guidelines for Americans, 2010. 7th Edition. US Government Printing Office; Washington, DC: 2010. Services.

6. Institute of Medicine Dietary Reference Intakes for Energy, Carbohydrate, Fiber, Fat, Fatty Acids, Cholesterol, Protein and Amino Acids (Macronutrients). National Academies Press; Washington, DC: 2002.

7. Institute of Medicine. Dietary reference intakes for calcium and vitamin D. The National Academies Press; Washington, DC: 2011.

8. Munoz KA, Krebs-Smith SM, Ballard-Barbash R, Cleveland LE. Food intakes of US children and adolescents compared with recommendations. Pediatrics. 1997; 100:323-329. [PubMed: 9282700]

9. Troiano RP, Briefel RR, Carroll MD, Bialostosky K. Energy and fat intakes of children and adolescents in the United States: Data from the National Health and Nutrition Examination Surveys. Am J Clin Nutr. 2000; 72(suppl 5):1343S-1353S. [PubMed: 11063476]

10. Neumark-Sztainer D, Story M, Hannan PJ, Croll J. Overweight status and eating patterns among adolescents: Where do youths stand in comparison to Healthy People 2010 objectives? Am J Public Health. 2002; 92:844-851. [PubMed: 11988458]

11. Mayer-Davis EJ, Nichols M, Liese AD, Bell RA, Dabelea DM, Johansen JM, Pi hoker C, Rodriguez BL, Thomas J, Williams D. for the SEARCH for Diabetes in Youth Study. Dietary Intake among youth with Diabetes: The SEARCH for Diabetes in Youth Study. J Amer Diet Assoc. 2006; 106(5):689-97. [PubMed: 16647326]

12. Gunther ALB, Liese AD, Bell RA, Dabelea D, Lawrence JM, Rodriguez BL, Standiford DA, Mayer-Davis EJ. Association between the dietary approaches o hypertension diet and hypertension in youth with diabetes mellitus. Hypertension. 2009; 53:6-12. [PubMed: 19029488] 
13. Liese AD, Bortsov A, Gunther ALB, Dabelea D, Reynolds K, Standiford DA, Liu L, Williams DE, Mayer-Davis EJ, D'Agnostino RB, Bell R, Marcovina S. Association of DASH diet with cardiovascular risk factors in youth with diabetes mellitus: The SEARCH for diabetes in youth study. Circulation. 2011; 123:1410-1417. [PubMed: 21422385]

14. Zeitler P, Epstein L, Grey M, Hirst K, Kaufman F, Tamborlane W, Wilfley D. Treatment options for type 2 diabetes in adolescents and youth: a study of the comparative efficacy of metformin alone or in combination with rosiglitazone or lifestyle intervention in adolescents with type 2 diabetes. Pediatr Diabetes. 2007:74-87. [PubMed: 17448130]

15. TODAY Study Group. Design of a family-based intervention for youth with type 2 diabetes: the TODAY study. Int J Obes. 2010; 34:217-226.

16. Grey M, Schreiner B, Pyle L. Development of a diabetes education program for youth with type 2 diabetes. Diabetes Educ. 2009; 35:108-116. [PubMed: 19244566]

17. Copeland KC, Zeitler P, Geffner M, et al. Characteristics of Adolescents and Youth with RecentOnset Type 2 Diabetes: The TODAY Cohort at Baseline. J Clin Endocrinol Metab. 2011; 96(1): 159-167. [PubMed: 20962021]

18. Breslow RA, Subar AF, Patterson BH, Block G. Trends in food intake: the 1987 and 1992 National Health Interview Surveys. Nutr Cancer. 1997; 28(1):86-92. [PubMed: 9200155]

19. Mayer-Davis EJ, Sparks KC, Hirst K, Costacou T, Lovejoy J, Regensteiner JG. Dietary intake in the Diabetes Prevention Program cohort: Baseline and 1-year post-randomization. Ann Epidemiol. 2004; 14:763-772. [PubMed: 15573453]

20. Vitolins MZ, Anderson AM, Delahanty L, Raynor H, Miller GD, Mobley C, Reeves R, Yamamoto M, Champagne C, Wing RR, Mayer-Davis E, Look AHEAD Research Group. Action for Health in Diabetes (Look AHEAD) trial: Baseline evaluation of selected nutrients and food group intake. J Am Diet Assoc. Aug; 2009 109(8):1367-1375. [PubMed: 19631042]

21. Hill RJ, Davies PS. The validity of self-reported energy intake as determined using doubly labeled water technique. Br J Nutr. 2011; 85(4):415-30. [PubMed: 11348556]

22. Block, G.; Murphy, M.; Roullet, J.; Wakimoto, P.; Crawford, P.; Block, T. Pilot validation of a FFQ for children 8-10 years; Paper presented at: Fourth International Conference on Dietary assessment Methods; Tucson, AZ. Septmeber 17, 2000;

23. Cullen KW, Watson K, Zakeri I. Relative reliability and validity of the Block Kids Questionnaire among youth aged 10-17 years. J Amer Diet Assoc. 2008; 108(5):862-66. [PubMed: 18442512]

24. Liese AD, D’Agostino RB Jr, Hamman RF, et al. SEARCH for Diabetes in Youth Study Group, The Burden of Diabetes Mellitus Among US Youth: Prevalence Estimates From the SEARCH for Diabetes in Youth Study. Pediatrics. 2006; 118:1510-1518. [PubMed: 17015542]

25. Lichtenstein AH, Appel LJ, Brands M, et al. Diet and lifestyle rcommendations revision 2006: A scientific statement from the American Heart Association Nutrition Committee. Circulation. 2006; 114:82-96. [PubMed: 16785338]

26. Nutrition and overweight. Healthy People 2010. 2nd ed.. US Government Printing Office; Washington, DC: 2000. p. 19-1-19-44.

27. The Surgeon General's Report on Nutrition and Health. US Public Service, Office of the Surgeon General; Washington, DC: 1988.

28. Sandler RB, Slemenda CW, Laporte RE, et al. Postmenopausal bone density and milk consumption in childhood and adlolescence. Am J Clin Nutr. 1985; 42:270-274. [PubMed: 3839625]

29. Aatola H, Koivistoinen T, Hutri-Kähönen N, et al. Lifetime fruit and vegetable consumption and arterial pulse wave velocity in adulthood: The Cardiovascular Risk in Young Finns Study. Circulation. 2010; 122:2521-2528. [PubMed: 21126970] 


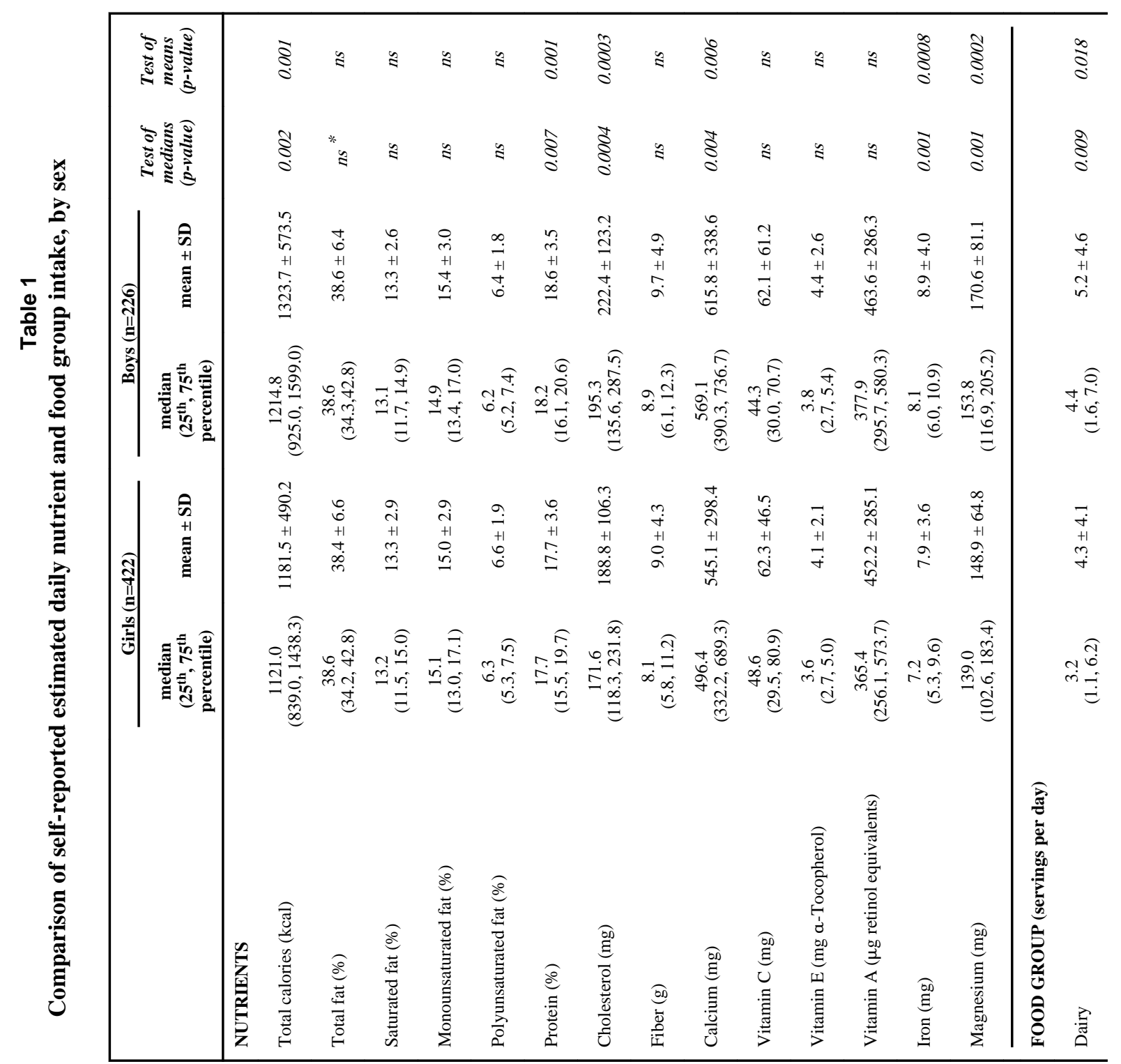




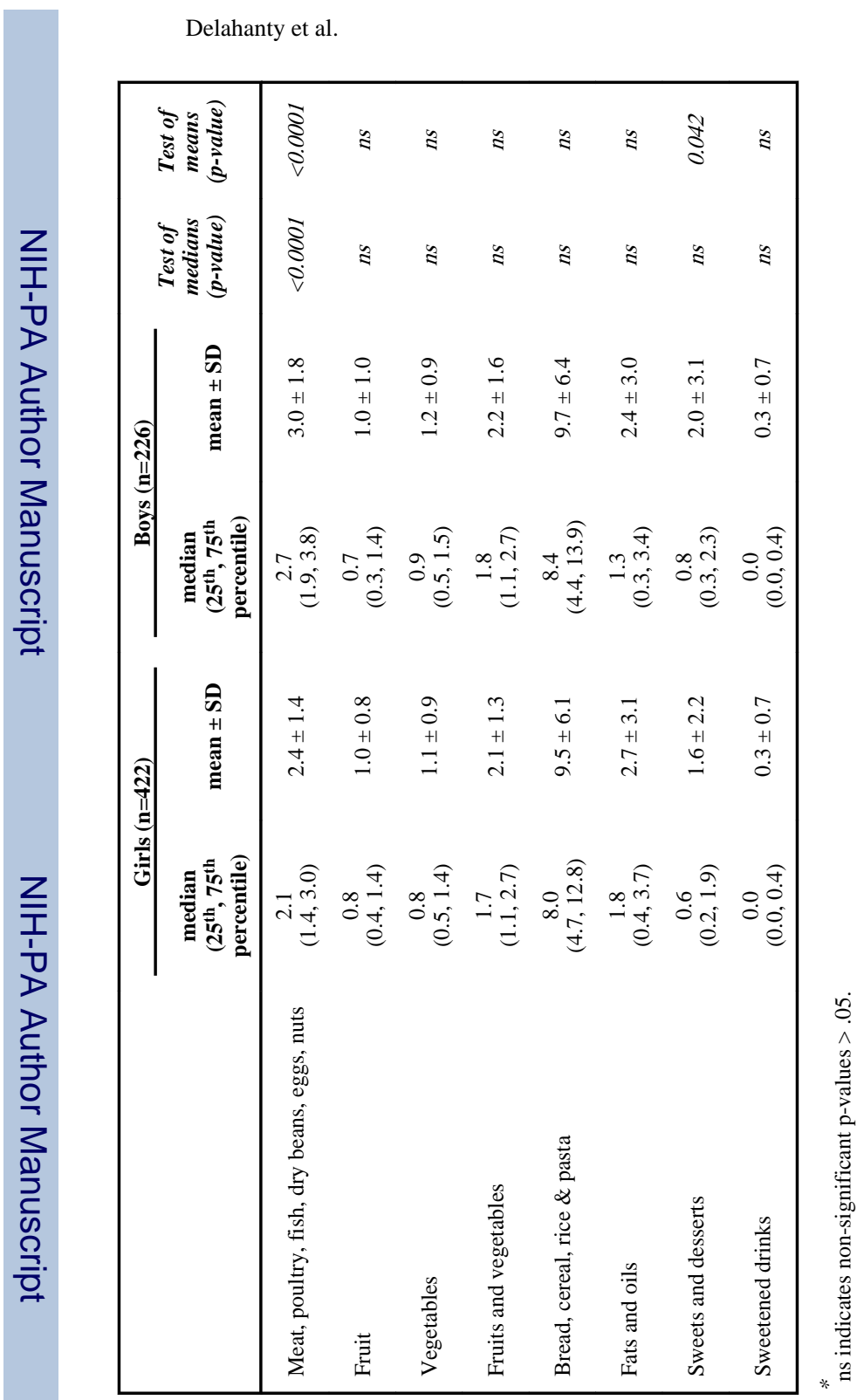




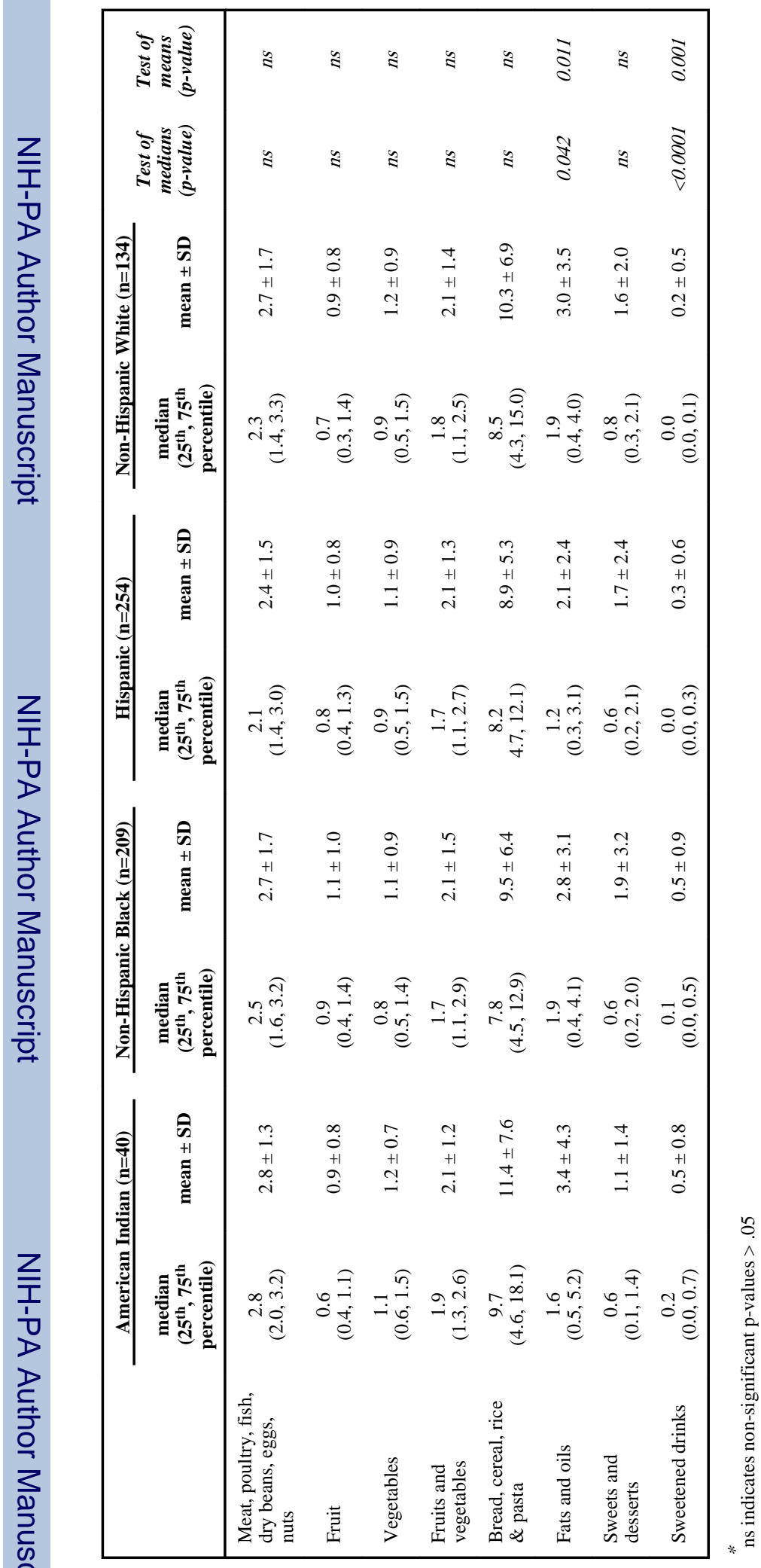




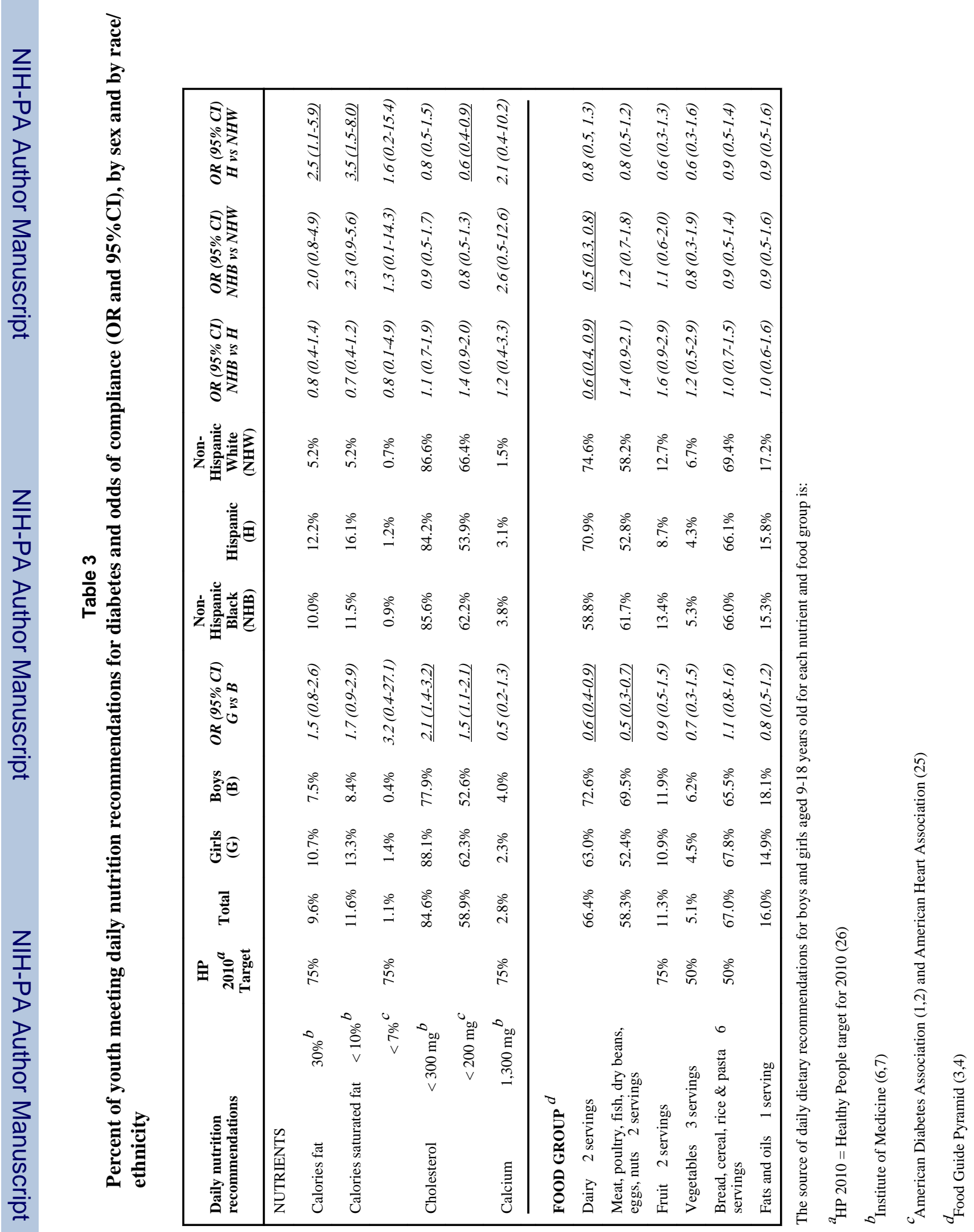

